\title{
Changes in ontogenetic patterns facilitate diversification in skull shape of Australian agamid lizards
}

\author{
Jaimi A. Gray ${ }^{1 *}$ D, Emma Sherratt ${ }^{1,2}$, Mark N. Hutchinson ${ }^{1,2}$ and Marc E. H. Jones ${ }^{1,2,3}$
}

\begin{abstract}
Background: Morphological diversity among closely related animals can be the result of differing growth patterns. The Australian radiation of agamid lizards (Amphibolurinae) exhibits great ecological and morphological diversity, which they have achieved on a continent-wide scale, in a relatively short period of time (30 million years). Amphibolurines therefore make an ideal study group for examining ontogenetic allometry. We used two-dimensional landmarkbased geometric morphometric methods to characterise the postnatal growth patterns in cranial shape of 18 species of amphibolurine lizards and investigate the associations between cranial morphology, and life habit and phylogeny.

Results: For most amphibolurine species, juveniles share a similar cranial phenotype, but by adulthood crania are more disparate in shape and occupy different sub-spaces of the total shape space. To achieve this disparity, crania do not follow a common post-natal growth pattern; there are differences among species in both the direction and magnitude of change in morphospace. We found that these growth patterns among the amphibolurines are significantly associated with ecological life habits. The clade Ctenophorus includes species that undergo small magnitudes of shape change during growth. They have dorsoventrally deep, blunt-snouted skulls (associated with terrestrial lifestyles), and also dorsoventrally shallow skulls (associated with saxicolous lifestyles). The sister clade to Ctenophorus, which includes the bearded dragon (Pogona), frill-neck lizard (Chlamydosaurus), and long-nosed dragon (Gowidon), exhibit broad and robust post-orbital regions and differing snout lengths (mainly associated with scansorial lifestyles).

Conclusions: Australian agamids show great variability in the timing of development and divergence of growth trajectories which results in a diversity of adult cranial shapes. Phylogenetic signal in cranial morphology appears to be largely overwritten by signals that reflect life habit. This knowledge about growth patterns and skull shape diversity in agamid lizards will be valuable for placing phylogenetic, functional and ecological studies in a morphological context.
\end{abstract}

Keywords: Agamidae, Evolutionary development, Geometric morphometrics, Lizards, Ontogeny, Skull

\section{Background}

The role of natural selection in producing morphological variation is limited by the changes that can be generated by organism-specific processes of growth and development (ontogeny) [1-4]. In each generation, the range of possible forms that natural selection can act upon is limited by the changes that can be generated by growth and development $[3,4]$. Throughout their development, organisms can undergo changes in shape, due to

\footnotetext{
*Correspondence: jaimi.gray@adelaide.edu.au

${ }^{1}$ School of Biological Sciences, University of Adelaide, Room 205E, Darling

Building North Terrace, Adelaide, SA 5005, Australia
}

Full list of author information is available at the end of the article differences in relative growth of components, and alterations in timing of their growth, a concept defined as ontogenetic allometry [3, 4]. Studies on ontogenetic allometry have been carried out since 1930, and considerable advances in methods have allowed exploration of patterns in more refined detail [4-10]. These studies have shown that changes in the attributes of ontogenetic patterns are important for facilitating evolutionary processes, $[4,7,11-13]$; evolutionary flexibility of ontogenies has been reported in several recent works e.g. [14-17].

There are two ways in which changes to an ancestral post-natal growth pathway can generate morphological diversity. Firstly, changes in adult shape can occur simply

(c) The Author(s). 2019 Open Access This article is distributed under the terms of the Creative Commons Attribution 4.0 International License (http://creativecommons.org/licenses/by/4.0/), which permits unrestricted use, distribution, and 
by changing adult size, without altering the shape-size relationship. Modifications in timing or rate of the ancestral growth pathway (heterochrony) account for diversification of shape. Such heterochronic changes, "when a descendant retains the ancestral relationship between size and shape" ([4] p. 87), correspond to predictions of the ontogenetic scaling hypothesis $[4,8]$. Secondly, changes in adult shape can occur due to departure from the ancestral growth pathway: changes to the relationship between size and shape (on a bivariate plot of size and shape, changes in slope, intercept, or a combination of both). This instance may be inferred when ontogenetic variation among members of a group does not map onto a common ontogenetic trajectory. Generally it was thought that growth pathways are phylogenetically stable, and that the ontogenetic scaling hypothesis can explain most variation $[18,19]$. However, growth pathways certainly can change; closely related taxa can show varying patterns of heterochrony $[14,20,21]$, and ontogenetic divergence [22] and convergence [23]. Variation in growth patterns among related taxa show that selection can rapidly modify postnatal developmental pathways under some circumstances (e.g. $[12-14,24])$.

Differences in ontogenetic patterns have often been associated with differences in ecology, in an interplay between selective forces and developmental processes. Evolutionary radiations consisting of many closely related species provide opportunities to examine how changes to an ancestral growth pathway may have contributed to morphological diversification within a particular clade. The Australian radiation of dragon lizards, the Amphibolurinae (Agamidae), includes iconic species such as the frill-neck lizard, bearded dragon, and thorny devil. They constitute a diverse component of Australia's reptile fauna comprising around 108 species, and probably represent the descendants of a single continental colonisation from Southeast Asia approximately 30 million years ago (Ma) [25-27]. They diversified into a range of distinct morphotypes and ecological niches as the continent became increasingly arid [28, 29], and today they are particularly diverse in the arid zone [30,31]. Among the most ecomorphologically relevant features of lizards is their head morphology (e.g. [32]); its role in supporting sensory structures, in food gathering, for social signalling and as a weapon, mean that it must be responsive to multiple selective pressures. Amphibolurinae includes some markedly varied and specialised skull shapes [33, 34], but apart from one recent limited study [35], there has been little examination of cranial growth patterns among different species.

This study aims to investigate whether the evolution of adult cranial shape diversity among 18 species of the Amphibolurinae is achieved through heterochrony alone, without changes to the ancestral growth pathway (as predicted by the ontogenetic scaling hypothesis), or through heterochrony and modification of these pathways. We use two-dimensional landmark based geometric morphometrics to characterise cranial shape, and compare the direction and magnitude of postnatal growth trajectories using a phenotypic trajectory analysis [36]. We also test for associations between ontogenetic patterns and life habit [37], and whether there is a phylogenetic signal in juvenile or adult skull shapes [38].

\section{Results}

\section{Variation in cranial shape}

A principal component analysis (PCA) characterising overall cranial shape across the sampled specimens shows that most of the smaller individuals have high $\mathrm{PC} 1$ values and low PC2 values (Fig. 1), which characterise skulls with relatively larger orbits and relatively shorter, smaller, and more slender post-orbit elements (jugal, postorbital, squamosal, parietal), and short blunt snouts. The other three quadrants of the morphospace are each associated with one of three major adult morphotypes. Gowidon longirostris is one example of an extreme morphotype (low PC1 values and low PC2 values) which has a relatively long and pointed snout, long maxillary facial process, a wide postorbital bar (jugal), small orbit, and an overall dorsoventrally shallow skull. Chlamydosaurus kingii, Intellagama lesueurii, Pogona barbata and $P$. vitticeps have relatively short snouts (compared to the most long snouted forms) and large, robust post-orbit elements (low PC1 values and high PC2 values). Interestingly, these four species are also those with the largest adult size. Ch. kingii and both species of Pogona have relatively short but pointed snouts, whereas I. lesueurii has a more rounded snout. The third extreme form of shape variation has a short and blunt snout, robust post-orbit elements, and a dorsoventrally deep overall skull profile, e.g. Ctenophorus nuchalis, Ct. reticulatus and Moloch horridus. For any particular species, the intermediate and adult ontogenetic stages occupy morphospace between the large-orbit form associated with smaller individuals (high PC1 values and low $\mathrm{PC} 2$ values), and one of these three broad morphotypes.

\section{Examining ontogenetic allometry}

Morphological disparity (Procrustes variance) for Amphibolurinae was significantly $(\boldsymbol{P}=\mathbf{0 . 0 0 1})$ greater among adult crania for each species (mean of the three largest specimens, Procrustes variance $=0.0148$ ) than among juvenile crania of each species (mean of the three smallest specimens, Procrustes variance $=0.0099$ ). The disparity calculated for the smallest juvenile and largest adult representatives of each species show that different agamid species begin life with a similar cranial shape and later disperse towards more disparate adult forms.

Tests for isometry in growth patterns for each species indicated that all species have significant allometric growth 


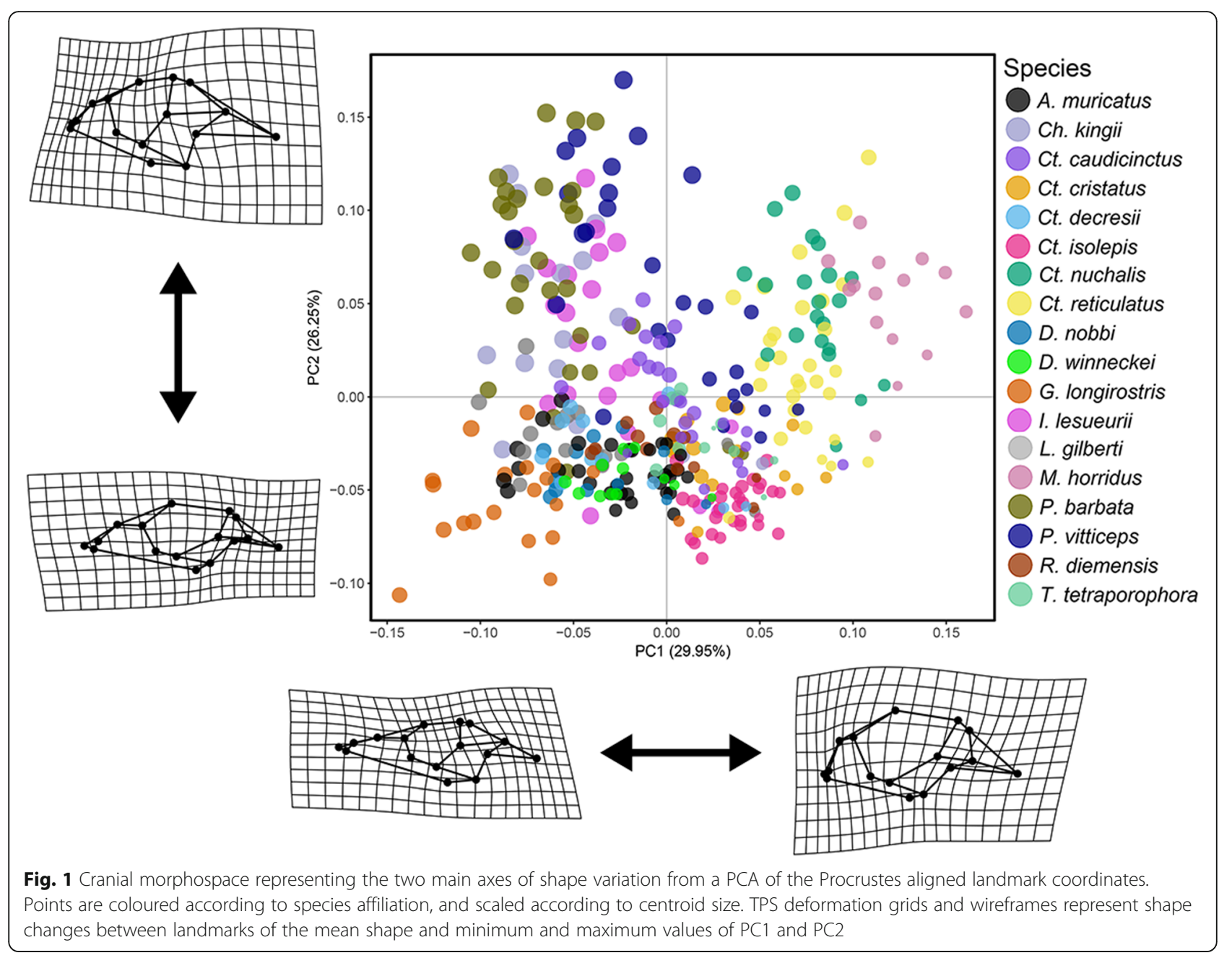

(lack of isometry). The variation detected in the shape data by a phenotypic trajectory analysis (PTA) revealed significant differences in growth trajectories: among directions (angles) of shape change, and also among magnitudes of shape change (Fig. 2a). For PC1, most species trajectories move from high values as juveniles, to low values as adults. For PC2, most species trajectories move from low values as juveniles, to high values as adults.

The direction of ontogenetic shape change varies between different species (Fig. 2a). Pairwise $P$-values for direction (angle) differences in the PTA are reported in Additional file 1: Table S2. Out of 153 possible pairs of species, 74 shared a common slope, while the remaining 79 pairs had significantly different directions of ontogenetic shape change. The pairwise results show that there are several cases where members belonging to the same clade or genus have different directions of ontogenetic shape change (e.g. Ctenophorus isolepis and Ct. nuchalis). $M$. horridus has a significantly different direction of shape change than all other sampled species. I. lesueurii, Ch. kingii, P. barbata, and P. vitticeps have the largest adult size, and all have similar directions of ontogenetic shape change. The species with the smallest adult sizes are $C t$. isolepis, Diporiphora winneckei, Rankinia diemensis, Tympanocryptis tetraporophora, and $M$. horridus, and they also have mostly similar directions of ontogenetic shape change, apart from $M$. horridus.

There are significant differences in the magnitudes of shape change between different species (Fig. 2a). Out of 153 possible pairs of species, 90 had a similar magnitude of shape change, while the remaining 63 pairs had significantly different magnitudes of shape change (Additional file 1: Table S3). Species in this study with a larger adult size (Ch. kingii, I. lesueurii, P. barbata, $P$. vitticeps) have greater magnitudes of ontogenetic shape changes than other sampled agamids. Ct. isolepis shows the smallest magnitude of shape change compared with all other sampled species. Ct. cristatus, $C t$. decresii, Ct. nuchalis and Ct. reticulatus all have relatively small magnitudes of shape change. In Ctenophorus, only three significant pairwise differences in magnitude were detected (both involving Ct. isolepis). 

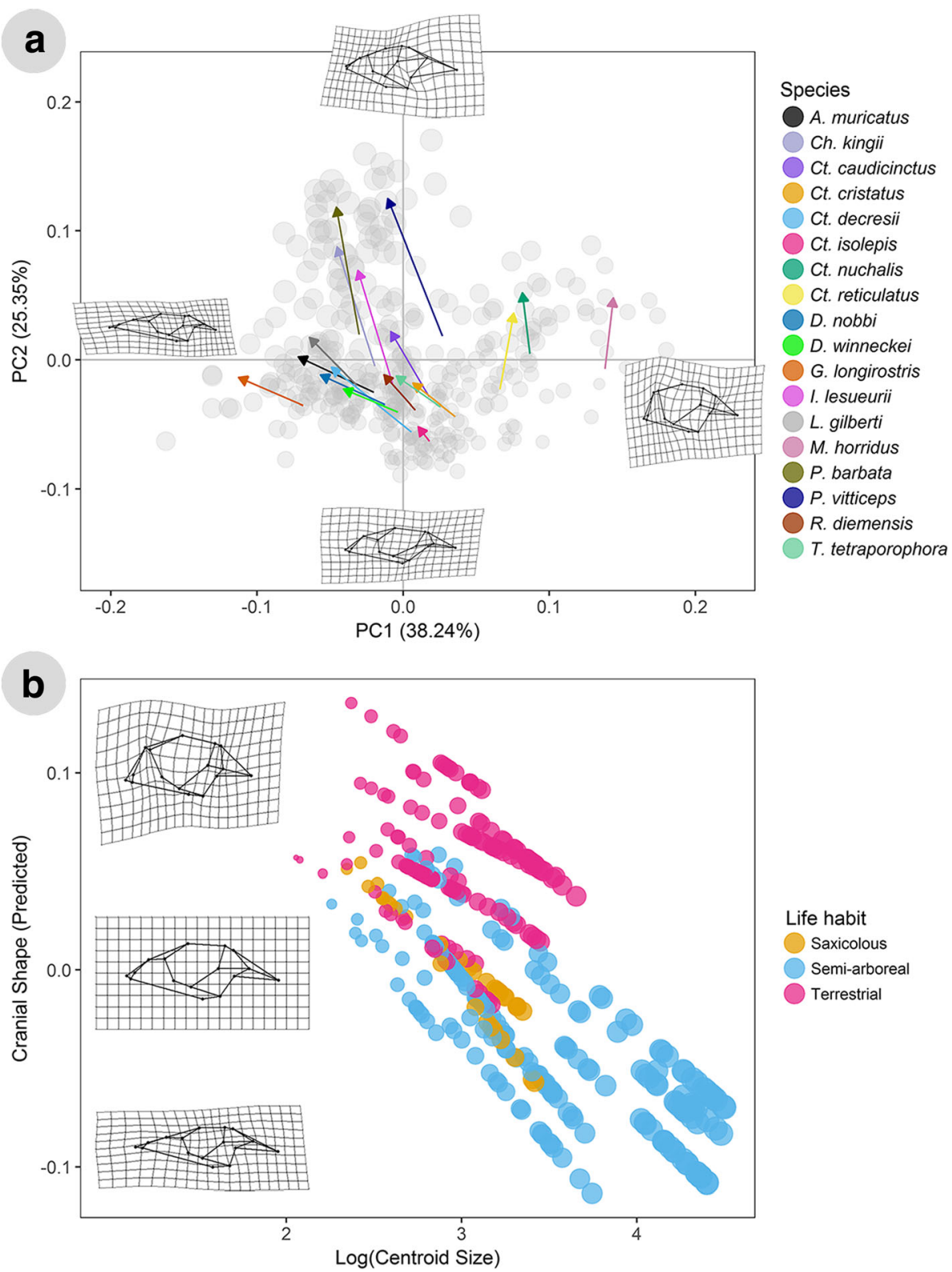

Fig. 2 Ontogenetic allometric trajectories derived from the phenotypic allometric trajectory analysis (a) and the species ontogenetic allometric patterns identified by life habit (b). For both plots, the size of points for each specimen is scaled to centroid size. In a, specimens are plotted on a morphospace represented by PCs 1 and 2 on the $x$ and y axes respectively. The arrows represent predicted trajectories for each species. Vectors represent ontogenetic trajectories of different species. The start of an arrow represents the mean juvenile shape and the end of the arrow represents the mean adult shape. The grey points represent the total variation within the sample. TPS deformation grids represent the shape change from the mean shape of the data set to the shape at the minimum and maximum values on that axis. In $b$, the $x$-axis represents logtransformed centroid size, and the $y$-axis represents the first principal component of the predicted values of multivariate regression of shape on size (as identified by MANCOVA). TPS deformations grids represent the shape change from the mean shape of the data set to the shape of the smallest and largest specimens in the data set

Within the sister clade to Ctenophorus we detected 21 pairwise differences, mostly involving Ch. kingii and the species of Pogona, which show the largest magnitude of shape change of any species (Additional file 1: Table S3).

Cranial shape is influenced by cranial size, life habit and the interactions of the two (MANCOVA, size $F_{(1,361)}=$
128.35, $\boldsymbol{P}=\mathbf{0 . 0 0 1}$; habit $\mathrm{F}_{(3,361)}=39.83, \boldsymbol{P}=\mathbf{0 . 0 0 1}$; size*habit $\left.\mathrm{F}_{(3,361)}=5.24, \boldsymbol{P}=\mathbf{0 . 0 0 1}\right)$. Thus, there is significant allometry in cranial shape, and the allometric slopes differ among life habit categories (see also Additional file 2: Table S4). The differences in ontogenetic allometric patterns (log transformed centroid size vs. predicted cranial shape) 
between species with different life habits is evident in Fig. 2b. Pairwise comparisons of allometric trajectories revealed that all life habit groups have significantly different slopes (direction of shape change) from one another, except for the semi-arboreal and saxicolous groups. There were also significant pairwise differences in trajectory length (amount of shape change) detected in pairwise comparisons for all three life habit groups (see Additional file 2: Table S5 for $P$-values for pairwise angle and length differences for life habit groups).

\section{Phylogenetic signal}

Tests for phylogenetic signal (relative to what is expected under a Brownian motion model of evolution) in cranial shape of the smallest juveniles and the largest adults revealed that both juveniles and adults show significant phylogenetic signal in their shape (juvenile: $\mathrm{K}_{\text {mult }}=0.44, \boldsymbol{P}=0.001$; adult: $\mathrm{K}_{\text {mult }}=0.44, \boldsymbol{P}=\mathbf{0 . 0 0 1}$ ). The amount of phylogenetic signal is less than 1 , indicating that species resemble each other less than is expected under Brownian motion. This is reflected in the dispersion of the species in the two cranial phylomorphospaces (Fig. 3), where there are many overlapping branches and where closely-related species are not adjacent in morphospace. The $K_{\text {mult }}$ estimates are the same for both the juvenile and adult shape data, despite the different dispersion patterns in the two phylomorphospaces. Conversely, there was no significant phylogenetic signal detected in cranial size (juveniles: $K=0.31, P=$ 0.130 ; adults, $K=0.36, P=0.063$ ).

\section{Discussion}

The amphibolurine post-natal growth pathway is evolutionarily flexible and has played a major role in producing great morphological disparity in adult cranial shape. Shape variation in both juveniles and adults had some phylogenetic signal, indicating that inheritance plays some role in structuring the morphological and ontogenetic variation we observe. However, different ontogenetic patterns are significantly associated with different life habits, suggesting that a significant amount of the variation in adult cranial shape is adaptive. Overall, it appears that environmental pressures often overwrite evolutionary history to influence ontogenetic patterns of skull shape in this radiation of lizards.

Most of the disparity in the adult cranial form of the sampled amphibolurines develops during post-hatching ontogeny. For just under half of the sampled species, we observe shape changes along a shared allometric slope, with indicates paedomorphy as a particular heterochronic pattern in a number of species of Ctenophorus. This finding is consistent with the ontogenetic scaling hypothesis (changes in the time or rate of development along the growth pathway). However, heterochrony is insufficient to explain the entirety of high morphological diversity of the amphibolurines. More often than not, we observe differences in both direction and magnitude of growth pathways between pairs of species, which suggests ontogenetic divergence has been a major factor in the evolution of the disparity seen in adult amphibolurine lizards.

The extent to which ontogenies are conserved during evolution has been a continued topic of controversy that has influenced the development of phylogenetic methods $[8,10,39,40]$; this study adds to the growing amount of evidence that morphological ontogenies are as prone to selection and evolutionary change as other aspects of morphology. It has been suggested that changes in the direction of allometric slopes are rare, since they represent costly alterations to growth dynamics [7-9], but our study adds to the growing literature that shows such changes are quite common [13, 14, 41]. The importance of evolving ontogenies in generating morphological diversity in amphibolurines resembles what has been shown in other reptiles [16], other vertebrates [42, 43], and even in plants [44]. In some cases, developmental pathways do represent an evolutionary constraint, canalising the phenotypic variation of species into particular portions of morphospace, as has been reported in lacertid lizards [45]. It seems that ontogenies are more flexible than previously thought, and can allow morphology to explore previously unoccupied areas of morphospace.

In both juvenile and adult skull shapes, phylogenetic affinity is less strongly correlated with shape than are associations with ecological groups. Although we find strong associations with ecological life habit, this does not preclude the existence of other many important factors, such as diet or patterns of interspecific selection. The weak phylogenetic signal we observe agrees with other studies on the skulls of reptiles [21, 46], mammals $[47,48]$, and fish [49], that have identified greater associations between morphology and diet [12, 47], feeding habits $[49,50]$, habitat $[24,51]$, and environmental factors $[49,52]$, than with phylogeny. In contrast, some studies confirm a strong phylogenetic signal in morphological variation, such that ecological correlations are not evident $[51,53,54]$ or have minimal effect [55]. Although adaptive factors and phylogeny both undoubtedly play a role in shaping morphological diversity, the extent of this role evidently differs amongst clades and should be assessed on a case-by-case basis.

Our findings add to the growing body of evidence $[5,12,36,37,56]$ that highlights the importance of using a rigorous quantitative framework to investigate the underlying basis of phenotypic variation. We have yet to investigate the influence of sexual dimorphism on amphibolurine lizards because of the lack of sex information for most of the museum specimens studied. Sexual size dimorphism has been recorded for at least one species in this study (see $[57,58]$ ), and therefore we cannot 


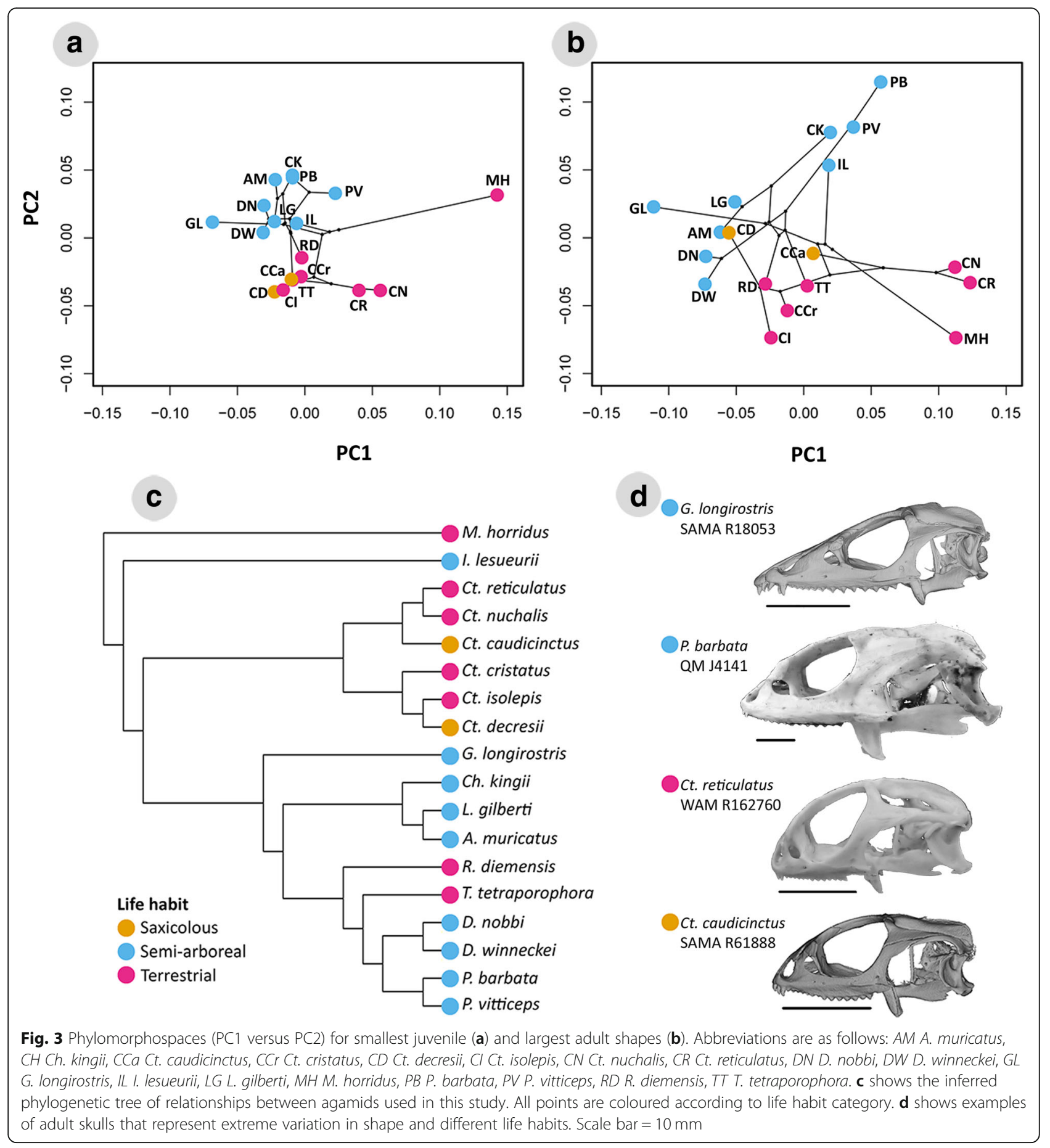

discount the possibility that it has an influence on skull shape, considering the strong allometric effects observed in these lizards. Therefore, future studies are encouraged to investigate the effect of sexual dimorphism on morphological variation in this clade. Furthermore, the influence on growth of diet and feeding habits on skull shape are yet to be studied in this group but are required to fully understand the evolutionary patterns we have observed. Our study thus serves as foundation for further studies to examine hypotheses about these factors and more.

\section{Conclusions}

Diversity in cranial shape of amphibolurine lizards seems to be the result of a combination of heterochrony and changes in growth patterns, which are related to phylogenetic affinity and adaptive evolution. The expectation 
of a conserved phylogenetic pattern, as predicted by the ontogenetic scaling hypothesis, does not fully explain the variation in skull shapes, and we find compelling evidence that there is an adaptive basis for much of the variation in ontogenetic allometry that we observe. Our study emphasises the power of growth pathways for facilitating the morphological variation that is characteristic of large and speciose evolutionary radiations. It also underlines the importance of using quantitative multivariate analyses to properly appreciate the role of developmental processes in shaping phenotypic diversity across species.

\section{Methods}

\section{Study specimens}

Material comprised 2D lateral view images of 361 specimens representing 18 different species of amphibolurine lizards (Table 1). The species were chosen to optimise taxonomic breadth, skull shape diversity, and size, but limited to species where the sample size was 10 specimens or more and included both juveniles and adults. We collected data from skeletal specimens from several institutions including South Australian Museum, University of Texas at Austin, Western Australian Museum, Field Museum of Natural History, Queensland Museum, University of Adelaide, and Melbourne Museum.

Table 1 Species studied

\begin{tabular}{llll}
\hline Species & $\mathrm{n}$ & $\begin{array}{l}\text { Average adult } \\
\text { skull length (mm) }\end{array}$ & Life habit \\
\hline Ctenophorus caudicinctus & 26 & 22.43 & Saxicolous \\
Ctenophorus cristatus & 15 & 24.39 & Terrestrial \\
Ctenophorus decresii & 10 & 23.51 & Saxicolous \\
Ctenophorus isolepis & 30 & 17.72 & Terrestrial \\
Ctenophorus nuchalis & 21 & 29.07 & Terrestrial \\
Ctenophorus reticulatus & 29 & 26.05 & Terrestrial \\
Amphibolurus muricatus & 34 & 28.72 & Semi-arboreal \\
Chlamydosaurus kingii & 17 & 75.97 & Semi-arboreal \\
Diporiphora nobbi & 12 & 23.22 & Semi-arboreal \\
Diporiphora winneckei & 12 & 13.37 & Semi-arboreal \\
Gowidon longirostris & 20 & 32.65 & Semi-arboreal \\
Lophognathus gilberti & 16 & 31.80 & Semi-arboreal \\
Pogona barbata & 29 & 64.83 & Semi-arboreal \\
Pogona vitticeps & 29 & 60.46 & Semi-arboreal \\
Rankinia diemensis & 12 & 18.97 & Terrestrial \\
Tympanocryptis tetraporophora & 11 & 15.93 & Terrestrial \\
Intellagama lesueurii & 22 & 70.96 & Semi-arboreal \\
Moloch horridus & 15 & 16.31 & Terrestrial \\
\hline Sample sizes were & & & Tvera
\end{tabular}

Sample sizes were dependent on availability from collections. Average skull length was calculated using the basal skull length of the largest three individuals of each species. Life habit categories were based on records in Wilson and Swan [65] and Cogger [66]

\section{Imaging}

The left side of the cranium was studied from 2D images. Each skeletal specimen was oriented using dry sand (black in colour for contrast) and photographed using an Olympus TG-4 camera mounted on a large flexible tripod, with a ruler set to the sagittal (midline) axis of the skull as a reference for scale. We also used 2D images of $3 \mathrm{D}$ rendered surface models generated from microComputed Tomography (CT) reconstructions of specimens in alcohol from South Australian Museum. These specimens had been micro CT scanned at either $\sim 18$ or $\sim 9 \mu \mathrm{m}$ resolution (depending on the size of the specimen) using a Skyscan 1076 (Bruker micro-CT) at Adelaide Microscopy. Each CT scan was reconstructed using the NRecon software interface [59]. We used Avizo v 9.0 [60] to digitally segment the cranium, threshold non-bone components from the scan, and render a surface model, which was then oriented laterally to capture a 2D image within Avizo, with a scale bar.

\section{Landmarks and shape analysis}

Lateral cranial shape was characterised using 2D landmark-based geometric morphometrics. Landmarks were digitised on the images of the crania using tpsDig v. 2.21 [40]. We set the scale for each specimen using scale bars present in the digital images, and digitised 16 single point landmarks (see Additional file 3: Figure S1), that represented equivalent points on bones at suture junctions, boundaries, and extremes of curvature on structures (see Additional file 4: Table S1, for landmark definitions). All subsequent analyses were performed using a routine written for the $\mathrm{R}$ statistical framework $\mathrm{v}$ 3.4.0. The raw 2D landmark coordinates were subjected to a generalised Procrustes alignment (GPA) using the $\mathrm{R}$ package geomorph [61]. This effectively removed differences in size, position, and orientation, leaving only shape variation [62]. The resulting Procrustes aligned shape coordinates were used as shape variables in subsequent analyses. Centroid size (the square root of the sum of the square distances of each landmark before GPA) was used as a proxy for body size. We were unable to use snout-vent length measurements because this data was not available for most of the skeletal specimens used.

\section{Visualising shape variation}

We performed a principal component analysis (PCA) on the Procrustes aligned shape coordinates to visualise the variation among sets of landmarks in the data set. To interpret the shape differences described by the major axes of variation identified by the PCA, we plotted a morphospace (PC1 versus $\mathrm{PC} 2$ ) with points identified by species and scaled to represent centroid size. To visualise the shape variation associated with the major axes of variation, we used thin-plate spline deformation grids [63], 
produced using the "plotRefToTarget" function in geomorph, and a wireframe representation of the skull, to represent shape differences between corresponding landmarks of the mean shape and minimum and maximum values for $\mathrm{PC} 1$ and $\mathrm{PC} 2$.

\section{Examining allometry}

To examine whether the morphological disparity in cranial shape among species differs between juveniles and adults, we quantified the disparity for two separate groups: the smallest three juveniles of each species (start of growth trajectory); and the largest three adults of each species (end of growth trajectory). We calculated morphological disparity using the "morphol.disparity" function in geomorph, which estimates Procrustes variance while accounting for group size, and uses absolute differences in variances to test for pairwise differences in morphological disparity between groups. The statistical significance between the juvenile and adult groups was assessed using a randomised residual permutation test with 1000 iterations.

We determined whether any species displayed isometric growth (no change in shape with a change in size), by fitting individual regression of log-transformed size on shape for each species, using the "procD.lm" function from the R package geomorph, which assesses significance via distributions generated with resampling permutations (we used 1000 iterations). If the association between size and shape for a particular species was significant, this result indicated that there was an ontogenetic allometric (and not isometric) pattern present for that particular species.

To test whether ontogenetic trajectories differ among species we conducted a phenotypic trajectory analysis (PTA) [64] on the shape coordinates using the "trajectory.analysis" function in geomorph. This procedure quantifies different attributes of a shape change trajectory between two or more points, in this case we measure the attributes of shape change between two groups: juveniles and adults. To circumvent issues with estimating nearness to adulthood in a clade with such broad variation in adult body sizes, we were able to categorise each specimen as either a juvenile or adult based on the number of acrodont teeth they had. If a specimen had more than $80 \%$ of the maximum number of acrodont teeth observed for that species, they were categorised as an adult. In some cases we altered the categorisation if a specimen was missing enough teeth to hinder obtaining a count, and were able to categorise these as either juveniles or adults based on the centroid sizes observed for other specimens of that particular species. We used species as groups, and juveniles and adults as the trajectory points. This analysis involved pairwise comparisons of two different attributes: the magnitudes of the trajectories among species, and also directions of the trajectories among species. Attribute differences were evaluated from sampling distributions generated from 1000 random permutations (based on a null model that lacked coefficients for a species-transect interaction) [36]. To visualise the ontogenetic phenotypic trajectories, we plotted the first two PCs of shape variation with arrows representing vectors of shape change (where the start of the arrows represents mean juvenile shapes and the end of the arrows represents mean adult shapes) and used thin-plate spline deformation grids to visualise shape change.

We tested for differences in ontogenetic allometric patterns of skull shape among ecological life habit groups by running a multivariate analysis of covariance (MANCOVA) model using the geomorph function "procD.allometry", with $\log$ transformed centroid size, life habit, and their interaction as model effects. Life habit was split into three categories (see Table 1), based on information available in Wilson and Swan [65] and Cogger [66]. Statistical significance was evaluated using Goodall's [67] F-ratio and a randomised residual permutation procedure using 1000 iterations [37]. If the interaction terms were significant, this indicated that the allometric trajectories differed among life habit groups. We identified which life habits groups differed from each other, using the "advanced.procD.lm" function in geomorph. These tests identified which life habit groups significantly differed in allometric slope from each other, via pairwise assessments of the similarity in slopes and intercepts through 1000 randomised residual permutations. To visualise ontogenetic allometric trajectories of species with different life habits, we plotted the predicted shape scores (from a multivariate regression of shape $\sim \log$ (size) "species), on log transformed centroid size, and identified points by life habit.

We inferred an evolutionary tree using Hugall et al., Melville et al., and Pyron et al. [25, 29, 68], and used this tree to estimate phylogenetic signal present in shape and size of the smallest juveniles and adults, relative to what is expected for the inferred phylogeny under a Brownian motion model of evolution. We used the mean shape of the smallest three individuals (by centroid size) for each species to estimate phylogenetic signal in juvenile shapes, and the mean of the largest three individuals (by centroid size) of each species to estimate phylogenetic signal in adult shapes. To estimate phylogenetic signal we calculated $K_{\text {mult }}$ [38], which is a generalisation of Blomberg's K-statistic appropriate for high-dimensional and multivariate data [69]. We determined statistical significance of $K_{\text {mult }}$ using phylogenetic permutation with 1000 iterations, which is calculated by permuting the multivariate shape data of the specimens among all tips of the phylogenetic tree. This was done using the "physignal" function in geomorph. To visualise how shape variation among species is associated with phylogeny we carried out separate PCAs on the landmark data for the mean shape of the three smallest juveniles 
and mean shape of the three largest adults, and used the phylomorphospace approach to project a phylogeny into the juvenile and adult PC biplots (with internal nodes estimated using maximum likelihood), implemented with the function "phylomorphospace" in the $\mathrm{R}$ package phytools [70].

\section{Additional files}

Additional file 1: Table S1. Pairwise results for angles in phenotypic trajectory analysis. Upper triangle shows pairwise $p$-values, while lower triangle shows pairwise angle differences (red highlighted cells indicate $p<0.05)$. Table S2. Pairwise results for magnitude in phenotypic trajectory analysis. Upper triangle shows pairwise $p$-values, while lower triangle shows pairwise magnitude differences (red highlighted cells indicate $p<0.05)$. (XLSX $19 \mathrm{~kb}$ )

Additional file 2: Table S3. Examining allometry: MANCOVAs of cranial shape predicted by size and life habit (shape $\sim \log ($ size)*habit). Table S4. Examining allometry of life habit groups: pairwise angle and length differences. Upper triangle $=p$-values. Lower triangle $=$ angles (DOCX $15 \mathrm{~kb}$ )

Additional file 3: Figure S1. Images of $A$. muricatus with landmark numbers which correspond to landmark definitions found in Additional file 4. (PPTX $2107 \mathrm{~kb}$ )

Additional file 4: Table S5. Landmark definitions used in geometric morphometric analysis (follows Evans 2008 nomenclature of skeletal elements). (DOCX $12 \mathrm{~kb}$ )

\section{Abbreviations}

2D: two-dimensional; 3D: three-dimensional; AM: A. muricatus; CCA: Ct. caudicinctus; CCR: Ct. cristatus; CD: Ct. decresii; $\mathrm{CH}$ : Ch. kingii; $\mathrm{Cl}$ : Ct. isolepis; CN: Ct. nuchalis; CR: Ct. reticulatus; CT: computed tomography; DN: D. nobbi DW: D. winneckei; GL: G. Iongirostris; GPA: Generalised Procrustes alignment; IL: I. lesueurii; LG: L. gilberti; MANCOVA: Multivariate analysis of covariance; MH: M. horridus; Ma: million years ago; PB: P. barbata; PC: Principal component; PCA: Principal component analysis; PTA: Phenotypic trajectory analysis; PV: P. vitticeps; RD: R. diemensis; TT: T. tetraporophora

\section{Acknowledgements}

We thank Chris Bell (University of Texas, Austin, USA); Alan Resetar (Field Museum, Chicago, USA), Scott Hocknull, Andrew Amey (Queensland Museum, Brisbane, Australia), Carolyn Kovach (South Australian Museum, Australia) for access to specimens. Ruth Williams (Adelaide Microscopy) and Amy Parker Watson (South Australian Museum), for assistance with CT scanning. Thanks also go to anonymous reviewers for critiques on this manuscript.

\section{Funding}

This study was conducted as part of JAG'S PhD thesis and was supported by an Australian Postgraduate scholarship, a Royal Society of South Australia grant, and a Jackson School of Geosciences travel grant. Australian Research Council grant (to MEHJ); Grant number: DE130101567 for CT scans and software. Support was also provided by South Australian Museum, the Environment Institute, and the University of Adelaide. The funding bodies played no role in design of the study, collection, analysis, or interpretation of data, or in writing the manuscript.

\section{Availability of data and materials}

The data sets supporting the results of this article are available in the additional files and in the MorphoBank repository. The link to the data in Morphobank repository will be provided upon reasonable request.

\section{Authors' contributions}

JAG, MNH, and MEHJ conceived of and designed the project. JAG collected, processed and analysed the data with essential statistical guidance provided by ES. JAG wrote the first draft of the manuscript and designed the figures. All authors contributed to and edited the final manuscript. All authors read and approved the final manuscript.
Ethics approval and consent to participate

Not applicable

\section{Consent for publication}

Not applicable

\section{Competing interests}

The authors declare that they have no competing interests.

\section{Publisher's Note}

Springer Nature remains neutral with regard to jurisdictional claims in published maps and institutional affiliations.

\section{Author details}

${ }^{1}$ School of Biological Sciences, University of Adelaide, Room 205E, Darling Building North Terrace, Adelaide, SA 5005, Australia. ${ }^{2}$ South Australian Museum, Adelaide, SA 5000, Australia. ${ }^{3}$ Earth Sciences, Natural History Museum, London SW7 5BD, UK.

Received: 30 April 2018 Accepted: 17 December 2018

Published online: 08 January 2019

\section{References}

1. Tokita M, Yano W, James HF, Abzhanov A. Cranial shape evolution in adaptive radiations of birds: comparative morphometrics of Darwin's finches and Hawaiian honeycreepers. Phil Trans R Soc B. 2017:372:20150481.

2. Losos JB. Lizards in an evolutionary tree: ecology and adaptive radiation of anoles, vol. 10. Oakland: University of California Press; 2011.

3. Klingenberg CP. Size, shape, and form: concepts of allometry in geometric morphometrics. Dev Genes Evol. 2016;226:1-25.

4. Klingenberg CP. Heterochrony and allometry: the analysis of evolutionary change in ontogeny. Biol Rev. 1998;73:79-123.

5. Klingenberg CP. Multivariate allometry. In: Marcus LF, Corti M, Loy A, Naylor GJP, Slice DE, editors. Advances in morphometrics. Boston: Springer US; 1996. p. 23-49.

6. Klingenberg CP, Marugán-Lobón J. Evolutionary covariation in geometric morphometric data: analyzing integration, modularity, and allometry in a phylogenetic context. Syst Biol. 2013;62:591-610.

7. Gould S. Allometry and size in ontogeny and phylogeny. Biol Rev. 1966;41: 587-640.

8. Gould SJ. Ontogeny and Phylogeny. Cambridge: Harvard University Press; 1977.

9. Huxley JS, Teissier G. Terminology of relative growth. Nature. 1936;114:895-6.

10. Nelson G. Outgroups and ontogeny. Cladistics. 1985;1:29-45.

11. Cock AG. Genetical aspects of metrical growth and form in animals. Q Rev Biol. 1966;41:131-90

12. Wilson $L A B$, Sánchez-Villagra MR. Diversity trends and their ontogenetic basis: an exploration of allometric disparity in rodents. Proc R Soc B. 2010;277:1227-34

13. Wilson $L A B$, Sánchez-Villagra MR. Evolution and phylogenetic signal of growth trajectories: the case of chelid turtles. J Exp Zool (Mol Dev Evol). 2011;316B:50-60.

14. Adams DC, Nistri A. Ontogenetic convergence and evolution of foot morphology in European cave salamanders (family: Plethodontidae). BMC Evol Biol. 2010;10:216.

15. Hugi J, Hutchinson MN, Koyabu D, Sánchez-Villagra MR. Heterochronic shifts in the ossification sequences of surface- and subsurface-dwelling skinks are correlated with the degree of limb reduction. Zoology. 2012;115:188-98.

16. Esquerré $\mathrm{D}$, Sherratt $\mathrm{E}$, Keogh JS. Evolution of extreme ontogenetic allometric diversity and heterochrony in pythons, a clade of giant and dwarf snakes. Evolution. 2017;71:2829-44.

17. Klingenberg CP. There's something afoot in the evolution of ontogenies. BMC Evol Biol. 2010;10:221

18. West GB, Brown JH. Enquist BJ. A general model for the origin of allometric scaling laws in biology. Science. 1997;276:122-6.

19. Gould SJ. The structure of evolutionary theory. Cambridge: Harvard University Press; 2002

20. Klingenberg CP, Zimmermann M. Static, ontogenetic and evolutionary allometry: a multivariate comparison in nine species of water striders. Am Nat. 1992;140:601-20.

21. Hipsley CA, Müller J. Developmental dynamics of ecomorphological convergence in a transcontinental lizard radiation. Evolution. 2017;71:936-48. 
22. Bastir M, Rosas A. Facial heights: evolutionary relevance of postnatal ontogeny for facial orientation and skull morphology in humans and chimpanzees. J Hum Evol. 2004;47:359-81.

23. Piras P, Colangelo P, Adams DC, Buscalioni A, Cubo J, Kotsakis T, Meloro C, Raia P. The Gavialis-Tomistoma debate: the contribution of skull ontogenetic allometry and growth trajectories to the study of crocodylian relationships. Evol Dev. 2010;12:568-79.

24. Urošević A, Ljubisavljević K, Ivanović A. Patterns of cranial ontogeny in lacertid lizards: morphological and allometric disparity. J Evol Biol. 2013;26:399-415.

25. Hugall AF, Foster R, Hutchinson M, Lee MSY. Phylogeny of Australian agamid lizards based on nuclear and mitchondrial genes: implications for morphological evolution and biogeography. Biol J Linnean Soc. 2008;93: 343-58.

26. Melville J, Ritchie EG, Chapple SNJ, Glor RE, Schulte JA. Evolutionary origins and diversification of dragon lizards in Australia's tropical savannas. Mol Phylogenet Evol. 2011:58:257-70

27. Oliver PM, Hugall AF. Phylogenetic evidence for mid-Cenozoic turnover of a diverse continental biota. Nat Ecol Evol. 2017;1:1896.

28. Fujioka T, Chappell J. History of Australian aridity: chronology in the evolution of arid landscapes. Geol Soc Spec Publ. 2010;346:121-39.

29. Melville J, Schulte JA. Larson a. a molecular phylogenetic study of ecological diversification in the Australian lizard genus Ctenophorus. J Exp Zool. 2001; 291:339-53.

30. Powney GD, Grenyer R, Orne CDL, Owens IPF, Meiri S. Hot, dry and different: Australian lizard richness is unlike that of mammals, amphibians and birds. Glob Ecol Biogeogr. 2010;19:386-96.

31. Melville J, Harmon LJ, Losos JB. Intercontinental community convergence of ecology and morphology in desert lizards. Proc R Soc B. 2006;273:557-63.

32. Kohlsdorf T, Grizante MB, Navas CA, Herrel A. Head shape evolution in Tropidurinae lizards: does locomotion constrain diet? J Evol Biol. 2008;21:781-90.

33. Bell CJ, Mead JI, Swift SL. Cranial osteology of Moloch horridus (Reptilia: Squamata: Agamidae). Rec West Aust Mus. 2009;25:201-37.

34. Siebenrock F. Das skelett der Agamidae. Sittzungsber Akad Wiss Wien 1895; 104

35. Stilson KT, Bell CJ, Mead Jl. Patterns of variation in the cranial osteology of three species of endemic Australian lizards (Ctenophorus: Squamata: Agamidae): implications for the fossil record and morphological analyses made with limited sample sizes. J Herpetol. 2017;51:316-29.

36. Collyer ML, Adams DC. Phenotypic trajectory analysis: comparison of shape change patterns in evolution and ecology. Hystrix. 2013;24:75-83.

37. Collyer ML, Sekora DJ. Adams DC. A method for analysis of phenotypic change for phenotypes described by high-dimensional data. Heredity. 2015;115:357-65.

38. Adams DC. A generalized K statistic for estimating phylogenetic signal from shape and other high dimensional multivariate data. Syst Biol. 2014;63:685-97.

39. Kluge AG, Strauss RE. Ontogeny and systematics. Ann Rev Ecol Syst. 1985;16: 247-68.

40. de Queiroz K. The ontogenetic method for determining character polarity and its relevance to phylogenetic systematics. Syst Zool. 1985;34:280-99.

41. Klingenberg CP. Evolution and development of shape: integrating quantitative approaches. Nat Rev Genet. 2010;11:623-35.

42. Weston EM. Evolution of ontogeny in the hippopotamus skull: using allometry to dissect developmental change. Biol J Linnean Soc. 2003:80:625-38

43. Wilson $L A B$. The evolution of ontogenetic allometries in mammalian domestication. Evolution. 2018;IN PRESS. https://doi.org/10.1111/evo.13464

44. Strelin MM, Benitez-Vieyra S, Fornoni J, Klingenberg CP, Cocucci AA. Exploring the ontogenetic scaling hypothesis during the diversification of pollination syndromes in Caiophora (Loasaceae, subfam. Loasoideae). Ann Bot. 2016:117:937-47.

45. Piras P, Salvi D, Ferrara G, Maiorino L, Delfino M, Pedde L, Kotsakis T. The role of post-natal ontogeny in the evolution of phenotypic diversity in Podarcis lizards. J Evol Biol. 2011;24:2705-20.

46. Claude J, Pritchard PCH, Tong H, Paradis E, Auffray J-C. Ecological correlates and evolutionary divergence in the skull of turtles: a geometric morphometric assessment. Syst Biol. 2004;53:933-48.

47. Fuchs $M$, Geiger M, Stange M, Sánchez-Villagra MR. Growth trajectories in the cave bear and its extant relatives: an examination of ontogenetic patterns in phylogeny. BMC Evol Biol. 2015;15:239.
48. Wilson LAB. The evolution of morphological diversity in rodents: patterns of cranial ontogeny. Doctoral Dissertation. Zurich: University of Zurich; 2010.

49. Frédérich $B$, Vandewalle P. Bipartite life cycle of coral reef fishes promotes increasing shape disparity of the head skeleton during ontogeny: an example from damselfishes (Pomacentridae). BMC Evol Biol. 2011;11:82.

50. Herrel A, Joachim R, Vanhooydonck B, Irschick DJ. Ecological consequences of ontogenetic changes in head shape and bite performance in the Jamaican lizard Anolis lineatopus. Biol J Linnean Soc. 2006;89:443-54.

51. Hipsley CA, Rentinck M-N, Rödel M-O, Müller J. Ontogenetic allometry constrains cranial shape of the head-first burrowing worm lizard Cynisca leucura (Squamata: Amphisbaenidae). J Morphol. 2016;277:1159-67.

52. Marcy AE, Hadly EA, Sherratt E, Garland K, Weisbecker V. Getting a head in hard soils: convergent skull evolution and divergent allometric patterns explain shape variation in a highly diverse genus of pocket gophers (Thomomys). BMC Evol Biol. 2016:16:207.

53. Doke D, Morey R, Dahanukar N, Padhye SM, Paripatyadar SV. Ontogenetic trajectory and allometry of Diplonychus rusticus (Fabricius), an oriental aquatic bug (Hemiptera: Belostomatidae) from the Western Ghats of India. Arthropod Struct Dev. 2017;46:297-303.

54. Dial TR, Reznick DN, Brainerd EL. Heterochrony in the evolution of Trinidadian guppy offspring size: maturation along a uniform ontogenetic trajectory. Proc R Soc B. 2017;284. Article number 20171319.

55. Powder KE, Milch K, Asselin G, Albertson RC. Constraint and diversification of developmental trajectories in cichlid facial morphologies. EvoDevo. 2015;6:25.

56. Wilson LB, Furrer $H$, Stockar R. Sanchez-Villagra MR. a quantitative evaluation of evolutionary patterns in opercle bone shape in Saurichthys (Actinopterygii: Saurichthyidae). Palaeontology. 2013;56:901-15.

57. Badham JA. The Amphibolurus barbatus species-group (Lacertilia: Agamidae). Aust J Zool. 1976;24:423-43.

58. Thompson G, Withers P. Size-free shape differences between male and female Western Australian dragon lizards (Agamidae). Amphibia-Reptilia. 2005;26:55-63.

59. Skyscan. NRecon. Aartselaar, Belgium. 2011.

60. Visualization Sciences Group. Avizo. Oregon: FEl Corporate Headquarters; 2013.

61. Adams DC. Otárola-Castillo E. geomorph: an R package for the collection and analysis of geometric morphometric shape data. Methods Ecol Evol. 2013:4:393-9.

62. Rohlf FJ, Slice DE. Extensions of the Procrustes method for the optimal superimposition of landmarks. Syst Zool. 1990:39:40-59.

63. Bookstein FL. Morphometric tools for landmark data: geometry and biology. Cambridge: Cambridge University Press; 1991.

64. Adams DC, Collyer ML. A general framework for the analysis of phenotypic trajectories in evolutionary studies. Evolution. 2009;63:1143-54.

65. Wilson S. Swan G. a complete guide to reptiles of Australia. 4th edn. Chatswood: new Holland: Publishers; 2013.

66. Cogger H. Reptiles and amphibians of Australia. 7th ed. Collingwood: CSIRO Publishing; 2014.

67. Goodall C. Procrustes methods in the statistical analysis of shape. J Royal Stat Soc B. 1991;53:285-339.

68. Pyron RA, Burbrink FT, Wiens JJ. A phylogeny and revised classification of Squamata, including 4161 species of lizards and snakes. BMC Evol Biol. 2013;13:1-53.

69. Blomberg SP, Garland TJ, Ives AR. Testing for phylogenetic signal in comparative data: behavioural traits are more labile. Evolution. 2003;57:717-45.

70. Revell $\amalg$. Phytools: an R package for phylogenetic comparative biology (and other things). Methods Ecol Evol. 2012;3:217-23.

\section{Ready to submit your research? Choose BMC and benefit from:}

- fast, convenient online submission

- thorough peer review by experienced researchers in your field

- rapid publication on acceptance

- support for research data, including large and complex data types

- gold Open Access which fosters wider collaboration and increased citations

- maximum visibility for your research: over $100 \mathrm{M}$ website views per year

At BMC, research is always in progress.

Learn more biomedcentral.com/submissions 